\title{
A Virtual Lab Model to Integrate Computer Networking Courses
}

\author{
Irfan Syamsuddin \\ Politeknik Negeri Ujung Pandang \\ Makassar, Indonesia \\ irfans@poliupg.ac.id
}

\begin{abstract}
This paper presents an effort to enhance teaching and learning processes of several Computer Networking courses at the Department of Computer and Networking Engineering, State Polytechnic of Ujung Pandang. The use of virtualization technique is proposed to deal with limited capacities of computer laboratory to serve various practical courses. Some simulation software is selected and also associated laboratory practice modules are documented. After conducting remastering technique in Linux environment, a novel Integrated Virtual Lab for Computer Networking courses is produced.
\end{abstract}

\section{Keywords—virtual lab; integrate computer; networking}

\section{INTRODUCTION}

The traditional approach to teaching such as lecturing and group discussion seems inadequate to cope with today's learning requirements in which students need more hands-on practices to get experiences by themselves. It applies in particular for computer and information technology related courses where many laboratory exercises are provided for students to obtain more knowledge and skills.

At the Department of Computer and Networking Engineering in the State Polytechnic of Ujung Pandang, there are at least four courses related to computer networking for undergraduate students which require extensive laboratory experiments. In fact, not only these classes need access to the laboratory, but other courses that also need extensive practical exercises such as web programming courses and database related courses. As a result, high usage, as well as errors and misconfigurations, usually happen to the laboratory which may prevent other class to use the laboratory for some time.

To deal with limited laboratory resources, laboratory staff handling and managing different demands by different courses every semester. Therefore, they proposed the virtualization technology to manage it. Virtualization technique in this study is particularly applied to enable some computer network simulations tools operating in as a virtualized networking laboratory.

This paper shares our experience of exploiting various Linux remastering techniques in integrating several computer network simulation programs and finally running it as a virtual machine. Also, lab modules based on lecture materials are then combined through Linux remastering application and eventually resulting a ready to execute the laboratory in the form of virtual machine. Therefore, such portable laboratory enables students to perform the hands-on exercise by following the modules as guidance based on their own pace.

The purpose of this project is to enhance the learning experience of our students through standardized teaching modules in a virtualized environment with easy maintenance and without demanding additional hardware.

\section{VIRTUALIZATION}

In the 1960s, the first introduction of virtualization technology took place by IBM. Virtualization technology enables to emulate operating systems on top of a physical machine or personal computer. The user can run and install applications over the virtual operating systems just like with the physical ones [1].

As depicted in figure 1 , the building block of a virtualization technology consists of typical physical machines which able to run particular operating systems termed as host operating systems. Virtual machine monitor (VMM) application exists on top of it to provides virtual media to run virtualized operating systems which is called guest operating systems [1].

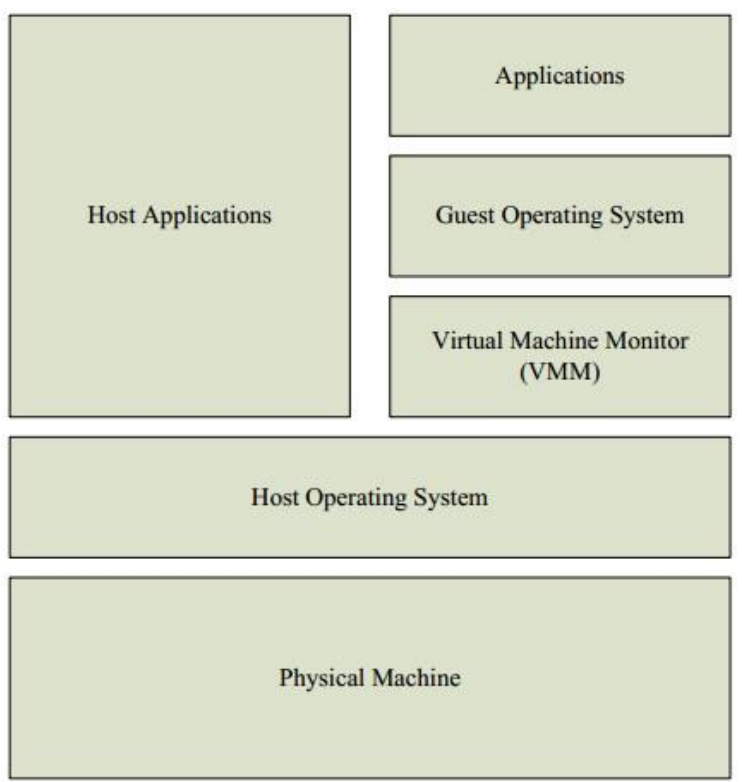

Fig. 1. Virtual machine concept 
Virtualization in the era of cloud computing has gained its popularity since it offers simplification of running and testing a new operating systems without disturbing host operating systems [2]. Also, the user may virtualize some operating systems simultaneously by establishing some virtual machines (VM) over a host physical machine.

\section{A. Mode of Virtual Machine Monitor}

The way VMM builds and controls the VM environment can be grouped into two approaches. The first way is called the full-virtualization mode in which VMs have no access to the host machine's resources while VMM simulates the functions of all these resources. The other way is called semivirtualization mode. In this manner, particular hardware parts in the host machines might be accessed by VMM [3].

There are advantages and disadvantages of both approaches. The main differences can be seen from the quality of service and simplicity of implementation. In one hand, fullvirtualization mode offers easier installation and maintenance, but its performance tends to be lower in comparison to semivirtualization. On the other hand, the application of semivirtualization mode usually shows higher performance indicator than the full-virtualization mode. Unfortunately, it is not easy to install and manage since it requires to make particular modifications in the guest OS [3].

\section{B. Virtualization Software}

Currently, there are several virtualization solutions produced for both personal computers (Windows and Linux) and Macintosh computers. The host operating system can be UNIX, FreeBSD, Linux, Mac OS $\mathrm{X}$ and Windows family (2000, XP, Vista, 2003, Windows 8 and Windows 10).

The commonly used Virtual Machine Monitor (VMM) programs include Microsoft Virtual Server, Parallels Workstation, QEMU, VMware, Xen and VirtualBox [1].

\section{ANALYSIS}

Considering its high performance and low cost of implementation, VirtualBox, the open source virtualization technology is preferred in this study. Once the virtualization technology applied, the following steps are made to achieve the goal of this study that is to establish a virtual networking lab.

The first one is mapping the courses that are related to computer networking. Five courses are selected, starting from the first year (i.e., Basic Computer Networking) to the final year (i.e., Network Security) courses. Each course uses different simulation software to assist students in achieving specific goals of each course.

Secondly, collecting related network simulation software. In this step, requirements of each software are identified to ensure minimum hardware requirements are achieved. The simulation software found and gathered at this stage are iNetwork [4], Packet Tracer [5], GNS3 [6] and Omnet++ [7]. All of them are combined and integrated into the next step.

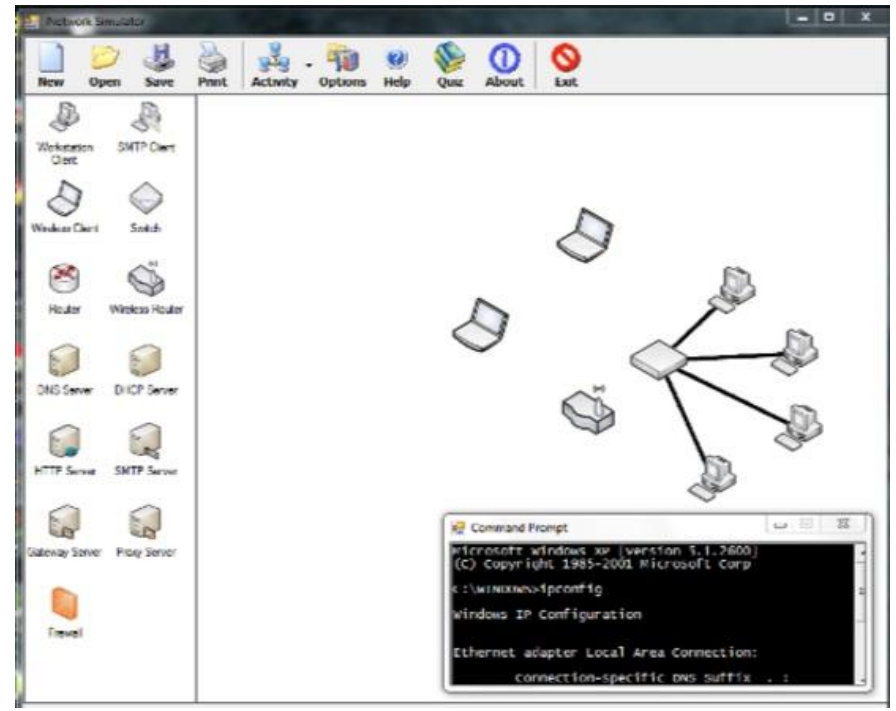

Fig. 2. iNetwork simulation software

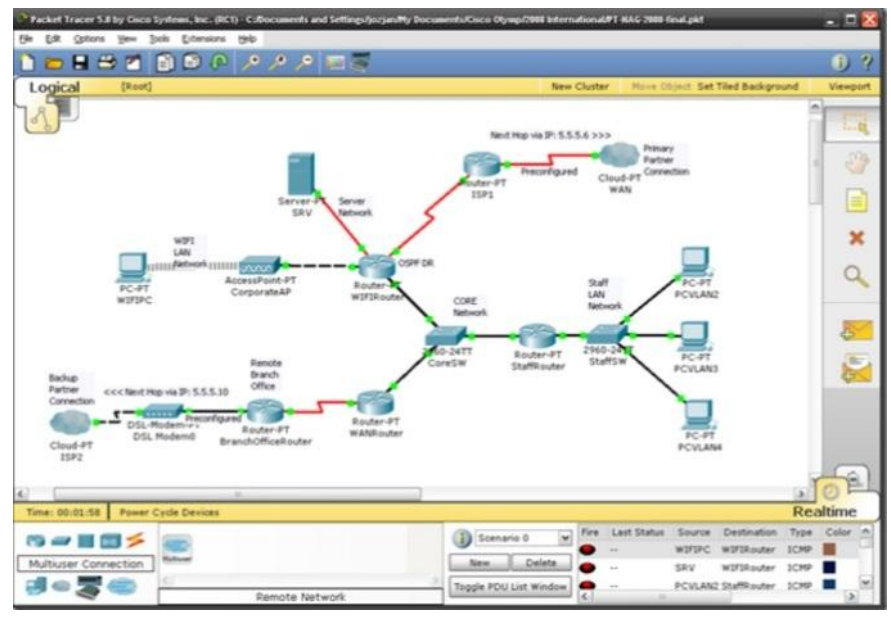

Fig. 3. PacketTracer simulation software

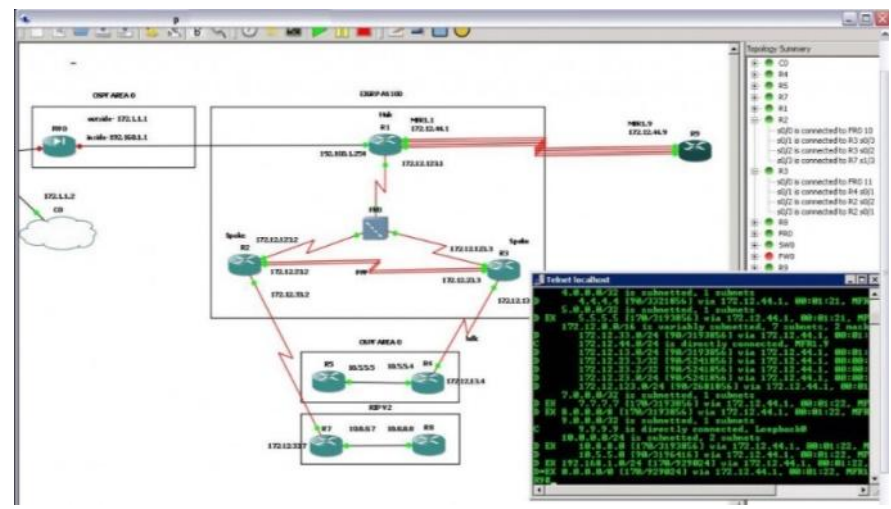

Fig. 4. GNS3 simulation software 


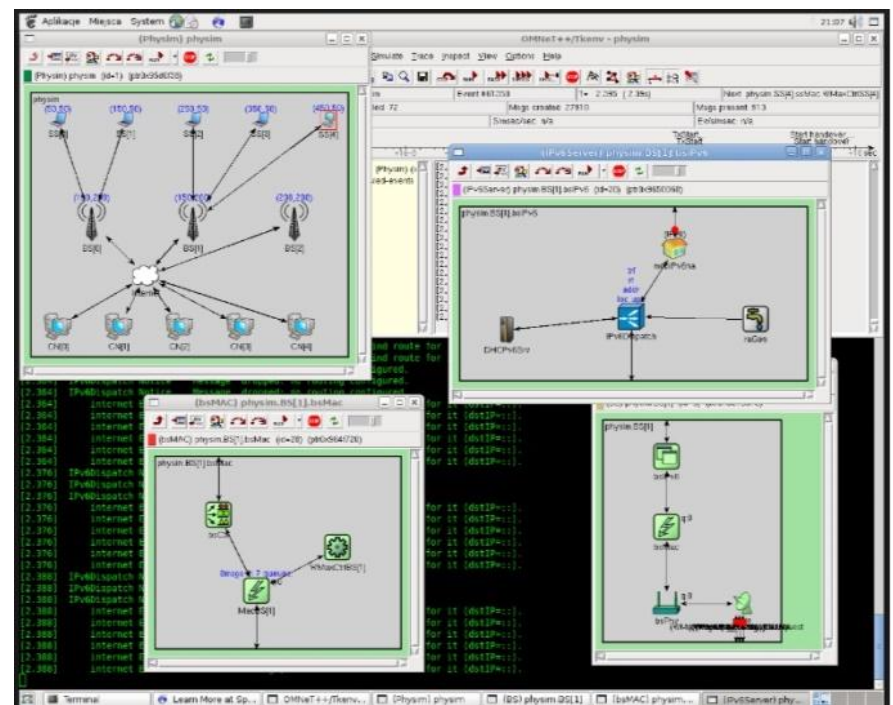

Fig. 5. Omnet++ simulation software

The next step is also called integration stage. This stage employs remastering technique in Linux distribution by removing unnecessary software packages and installing required simulation software such as iNetwork, PacketTracer, GNS3, and Omnet++. Also, supporting laboratory practice guidelines and related documents are packed according to associated courses. The output of this stage is a novel Integrated Virtual Lab for Computer Networking courses in the form of a virtual machine (VM).

Finally, the last stage is to perform testing and evaluation to the virtual machine as a living lab. This final step is necessary to identify any possible bugs or errors at an earlier stage before applying it.

\section{CONCLUSION}

In this paper, an effort to improve learning processes by employing virtualization technique is proposed to develop a novel Integrated Virtual Lab for Computer Networking courses. A four step approach is presented to describe the whole processes behind the production of the virtual machine.

In the future, this study will be extended in two ways. Firstly, improving the quality of service by conducting additional performance testing and evaluations of the VM in different scenarios. Secondly by measuring lecturers or students perceptions in using the virtual lab.

\section{References}

[1] P. Li and T. Mohammed, "Integration of virtualization technology into network security laboratory," in Frontiers in Education Conference, 2008. FIE 2008. 38th Annual, 2008, p. S2A-7.

[2] I. Syamsuddin, "Problem Based Learning on Cloud Economics Analysis Using Open Source Simulation.," Int. J. Online Eng., vol. 12, no. 6, 2016.

[3] P. Li, P. Lunsford, T. Mohammed, L. Toderick, and C. Li, "Using Virtual Machine Technology in an Undergraduate Intrusion Detection Lab," in Proceedings of 2007 ASEE Annual Conference and Exposition, 2007.

[4] M. Ye and K. Sandrasegaran, "Teaching about Firewall Concepts using the iNetwork Simulator," in Information Technology Based Higher Education and Training, 2006. ITHET'06. 7th International Conference on, 2006, pp. 889-892.

[5] J. Janitor, F. Jakab, and K. Kniewald, "Visual learning tools for teaching/learning computer networks: Cisco networking academy and packet tracer," in Networking and Services (ICNS), 2010 Sixth International Conference on, 2010, pp. 351-355.

[6] C. Welsh, GNS3 network simulation guide. Packt Publ., 2013.

[7] J.-L. Kuo, C.-H. Shih, and Y.-C. Chen, "Performance analysis of realtime streaming under TCP and UDP in VANET via OMNET," in ITS Telecommunications (ITST), 2013 13th International Conference on, 2013, pp. 116-121. 